\title{
Joyce invariants for K3 surfaces and mock theta functions
}

\author{
Anton Mellit and So OKada
}

For stability conditions on K3 surfaces, we study moduli stacks of semistable objects with Donaldson-Thomas type invariants, introduced by Joyce, and mock theta functions, introduced by Ramanujan. In particular, we will show invariance of moduli stacks on faithful stability conditions and motivic invariants, and in terms of mock theta functions, study generating functions obtained by moduli-stack counting and differential equations.

\section{Introduction}

\subsection{Stability conditions for K3 surfaces and Joyce invariants}

On triangulated categories, Bridgeland [4] introduced the notion of stability conditions, which extends standard stabilities such as Gieseker stabilities on the abelian category of coherent sheaves of a variety $X$, denoted by $\operatorname{Coh} X$, to the bounded derived category of $\operatorname{Coh} X$, denoted by $\mathrm{D}(X)$.

One way to think of the notion is that it is a tool to study interesting invariants on moduli stacks as we have seen in the fundamental work [14], in which the notion of Harder-Narasimhan filtrations was given birth to discuss Tamagawa numbers that are certain volumes of moduli spaces on curves.

As a background, let us recall that Douglas' work [11] on П-stabilities in superstring theory motivated the notion of stability conditions. In a CalabiYau variety $X$, boundaries of open strings restrict to subvarieties called B-branes. With Kontsevich's framework [21], in $\mathrm{D}(X)$, the notion of $\Pi$ stabilities discusses configuration of B-branes and its deformation, which is locally parametrized by central charges of B-branes. People have studied modular forms coming from physics such as conformal field theories. We count Bogomol'nyi-Prasad-Sommerfield (BPS) B-branes whose central charges align in the complex plane, and construct something close to modular forms (see [12, Section 6]).

We begin to be specific for this paper, leaving formality a bit out for later sections. For stability conditions of triangulated categories, Joyce started 
to extend Donaldson-Thomas invariants so that wall-crossings of stability conditions give differential equations over his invariants, which we call Joyce invariants.

An essential notion to define Joyce invariants is motivic invariants [16], which is explained as follows. Let us recall an algebraic group $G$ is called special $[10]$ if $\mathrm{G}$ is a group embedded in some $\mathrm{GL}(n, \mathbb{C})$ with $\mathrm{GL}(n, \mathbb{C}) \rightarrow$ $\mathrm{GL}(n, \mathbb{C}) / G$ being locally trivial. Then, from Artin stacks of finite type with affine geometric stabilizers, which, by Kresch [22], can be reduced to have stratification locally of type $[U / G]$ for quasiprojective varieties $U$ and special algebraic groups $G$, to a commutative $\mathbb{Q}$-algebra $\Lambda$ containing a variable $l$, each motivic invariant $I$ satisfies the following. For $I(\mathbb{C})=l, I(\mathrm{GL}(n, \mathbb{C}))=$ $l^{n^{2}}\left(1-l^{-1}\right) \ldots\left(1-l^{-n}\right)$ is invertible in $\Lambda$, for quasiprojective varieties $U$ and $V, \quad I(U \times V)=I(U) I(V)$, for a closed quasiprojective variety $V$ in $U, \quad I(U)=I(V)+I(U \backslash V)$ and for a quotient stack $[U / G]$ with a special algebraic group $G, I([U / G])=I(U) / I(G)$. For example, a motivic invariant $I$ can be taken to be virtual Hodge polynomials, generalized through the last condition.

From now on, $X$ denotes an algebraic K3 surface and, in the stability manifold of $X, \operatorname{Stab}^{*}(X)$ denotes the connected component constructed by Bridgeland [3]. In [15], on $\operatorname{Coh} X$, Joyce proved that his invariants exist independently of the choice of Gieseker stabilities; then, on $\mathrm{D}(X)$, he continued to discuss his invariants, supposing that his invariants exist independently of the choice of stability conditions in $\operatorname{Stab}^{*}(X)$, which was proved by Toda [31]. For a stability $\sigma$ of Gieseker on $\operatorname{Coh} X$ or of Bridgeland on $\mathrm{D}(X)$ and Mukai vectors $\alpha$ in the Mukai lattice of $X$ [23], let $M^{\alpha}(\sigma)$ be moduli stacks of semistable objects; Joyce invariants $J^{\alpha}(\sigma)$ are defined with motivic invariants on these moduli stacks (see Section 2.2).

With the notion of numerically faithfulness (faithful for short), which was introduced by Okada [26] (see Section 2.1), for each moduli stack, the independence of the choice of stability conditions in $\operatorname{Stab}^{*}(X)$ on Joyce invariants manifests itself as follows.

Theorem 1.1. For each algebraic K3 surface $X$, Mukai vector $\alpha$ of $X$, motivic invariant $I$, faithful stability conditions $\sigma, \sigma^{\prime}$ in $\operatorname{Stab}^{*}(X)$, we have $I\left(M^{\alpha}(\sigma)\right)=I\left(M^{\alpha}\left(\sigma^{\prime}\right)\right)$.

By [26], faithful stability conditions exist as a dense subset in $\operatorname{Stab}^{*}(X)$. By wall structures examined in [3], for each Mukai vector $\alpha$, polarization of $X$ and some faithful stability condition $\sigma$ in $\operatorname{Stab}^{*}(X), M^{\alpha}(\sigma)$ consists of Gieseker semistable coherent sheaves. 
For other Calabi-Yau surfaces such as abelian surfaces and minimal resolutions of surface singularities, one can check that Theorem 1.1 holds on their stability manifolds that have been studied (see [5]). Also, for some moduli stacks of stable objects and moduli stacks of $\mu$-semistable coherent sheaves on $X$, one can compare Theorem 1.1 with a sequence of flops in [1] and dimension counting in [32].

To make explicit computation of motivic invariants, we first want to know our moduli stacks as moduli spaces and compute stabilizer groups of objects. For example, for primitive Mukai vectors of positive ranks, by [32], moduli spaces of Gieseker stable coherent sheaves are deformation equivalent to Hilbert schemes with $\mathbb{C}^{*}$ stabilizer group for each point.

Going beyond above primitive cases is challenging; in general, moduli spaces can be singular and computing stabilizer groups is demanding. On the other hand, let us recall that for Mukai vectors $[E],[F]$ of objects $E, F$, Mukai parings are defined as $[E] .[F]=\sum_{i}(-1)^{i} \operatorname{dim} \operatorname{Ext}^{i}(E, F)$; then, for Mukai vectors $v$ with $v . v>0$, Theorem 1.1 boils down to Corollary 1.1.

Let us recall that Mukai vectors $\alpha$ with $\alpha . \alpha=2$ are called spherical; namely, $\alpha$ correspond to spherical objects, which give rise to autoequivalences of $\mathrm{D}(X)$ [30], such as structure sheaves supported over rational curves on $X$ and the structure sheaf of $X$. Notice that a Mukai vector $v$ with $v \cdot v>0$ is a multiple of spherical Mukai vector.

Corollary 1.1. For each spherical Mukai vector $\alpha$, faithful stability condition $\sigma$ in $\operatorname{Stab}^{*}(X)$ and positive integer $n$, we have $M^{n \alpha}(\sigma) \cong\left[\frac{1}{\operatorname{GL}(n, \mathbb{C})}\right]$.

Once we know moduli stacks in this detail of Corollary 1.1, we can compute various invariants. Indeed, after Okada discussed some part of this paper such as Corollary 1.2 (in the original form of Joyce invariants for some $\alpha$ ) in [25] and while the authors were preparing this paper, they got notified that, in [24], for standard stabilities of coherent sheaves of rational elliptic surfaces, Nakajima-Yoshioka computed their invariants. Also, for stability conditions of Calabi-Yau categories of dimension three, KontsevichSoibelman discussed their invariants [20].

We will stick to Joyce invariants for K3 surfaces. For the convenience of our formulas, we will use $q=l^{-1}$, switching between Tate motive and Lefschetz motive. Then, for cases in Corollary 1.1, we have the following.

Corollary 1.2. $J^{n \alpha}(\sigma)=\frac{q^{n^{2}}}{n\left(1-q^{n}\right)}$.

We would like to mention that we are slightly modifying the original formulation of Joyce invariants for K3 surfaces, as suggested to the authors 
by Zagier. Namely, in order to obtain more natural expressions, we omit the factor $\left(q^{-1}-1\right)=(l-1)$ in [15]; recall that the factor was involved so that we get numbers on moduli stacks of stable objects by replacing $q$ by one. Instead, we take residues at $q=1$ to extend Euler characteristics to moduli stacks, which are not necessarily only of stable objects. In particular, for cases in Corollary 1.2, we obtain $\frac{1}{n^{2}}$, which appear in the multiple cover formula of the disc [19], as pointed out to the authors by Diaconescu.

\subsection{Joyce invariants and mock theta functions}

Invariants on the moduli stacks have been studied in Bridgeland-ToledanoLaredo [2] and Kontsevich-Soibelman [20]. Central charges of stability conditions in $\operatorname{Stab}^{*}(X)$ factor through Mukai lattices. For $k \in \mathbb{Z}$, we would like to study the following generating functions of Joyce invariants in Corollary 1.2:

$$
J_{k}=\sum_{n>0} n^{k} J^{n \alpha}(\sigma)=\sum_{n>0} \frac{n^{k-1} q^{n^{2}}}{1-q^{n}} .
$$

Let us point out that taking residues termwise at $q=1$ gives $-\zeta(2-k)$.

These $J_{k}$ appear in the following sum suggested by Joyce [17]; namely, on stability manifolds, for Mukai vectors $v$ such that we have semistable objects $E$ with $[E]=v$, we can consider the sum $\sum_{v} Z(v)^{k} J^{v}(\sigma)$, which is invariant under autoequivalences. For spherical Mukai vectors $\alpha, \sum_{\alpha, n>0}$ $Z(n \alpha)^{k} J^{n \alpha}(\sigma)$ is again invariant under autoequivalences and we study its building piece.

Let us start from the following observation. Now, $J_{k}$ are already some quantum polylogarithms, as they are $q$-deformations of polylogarithms. However, the presence of $q^{n^{2}}$ in the numerator does not make, in particular, $J_{0}$ the well-known quantum dilogarithm (see [34]), but instead $J_{k}$ look similar to some of mock theta functions, which were introduced by Ramanujan $[27,28]$.

The theory of mock theta functions based on Zwegers' celebrated thesis [35], is now widely accepted in number theory [6-9,33]. In short, Zwegers provided a way to add correction terms to make mock theta functions into certain real analytic modular forms [33]. Let us briefly recall some notions as follows.

For $\tau$ in $q=\mathrm{e}^{2 \pi \mathrm{i} \tau}$, let $D=\frac{1}{2 \pi \mathrm{i}} \frac{d}{d \tau}$ and $\bar{D}=\frac{1}{2 \pi \mathrm{i}} \frac{d}{d \tau}, M^{k}$ the space of meromorphic modular forms of weight $k$ for $k \in \frac{1}{2} \mathbb{Z}$ with poles only at cusps, and $\tau=x+\mathrm{i} y$. Applying $\bar{D}$ on mock theta functions, we receive the notion called shadows [33], which lie in $\frac{M^{k^{\prime}} \otimes \bar{M}^{2-k}}{y^{k}}$ for $k^{\prime}, k \in \frac{1}{2} \mathbb{Z}$. For holomorphic 
functions $a(\tau)$ and $b(\tau)$, a canonical solution to $\bar{D}\left(R\left(\frac{a(\tau) \overline{b(\tau)}}{y^{k}}\right)\right)=\frac{a(\tau) \overline{b(\tau)}}{y^{k}}$ is the following (whenever it converges):

$$
R\left(\frac{a(\tau) \overline{b(\tau)}}{y^{k}} ; \tau\right):=2 \pi \mathrm{i} a(\tau) \overline{\int_{\mathrm{i} \infty}^{\tau} \frac{b(z) d z}{\left(-\frac{\mathrm{i}}{2}(z-\bar{\tau})\right)^{k}}}
$$

For a mock theta function $g(\tau), g(\tau)-R(\bar{D}(g(\tau))$ is the holomorphic part for the correction term $R(\bar{D}(g(\tau))){ }^{1}$

Going back to $J_{k}$, with certain duality, we would like to compensate our choices of $k$. This can be done in terms of differential equations, modular forms and certain correction terms to $J_{k}$. Here, for Eisenstein series and Bernoulli numbers $E_{k}$ and $B_{k}$, we notice the following relation between $J_{2-k}$ and $J_{k}$.

$$
D^{k-1} J_{2-k}=\frac{B_{k}}{2 k}\left(1-E_{k}\right)-J_{k}+\sum_{n>0} \frac{q^{n^{2}}}{n^{1-k}} \quad(k \geq 2) .
$$

We would like to have a duality formula only with modular forms. Let us recall that in the space of modular forms of a given degree, Eisenstein series make distinguished basis of the subspace that is orthogonal to cusp forms. Let us take the following.

\section{Definition 1.1.}

$$
\mathcal{J}_{k}=-\frac{B_{k}}{2 k}-\frac{1}{2} \sum_{n>0} n^{k-1} q^{n^{2}}+J_{k}=-\frac{B_{k}}{2 k}+\frac{1}{2} \sum_{n \neq 0} \frac{n^{k-1} q^{n^{2}}}{1-q^{n}} .
$$

This time, for positive even integers $k$, we have the following: ${ }^{2}$

$$
D^{k-1} \mathcal{J}_{2-k}+\mathcal{J}_{k}=-\frac{B_{k}}{2 k} E_{k}
$$

In this paper, we study $\mathcal{J}_{2}$, which reads

$$
\mathcal{J}_{2}(\tau)=-\frac{1}{24}-\frac{1}{2} \sum_{n>0} n q^{n^{2}}+J_{2}=-\frac{1}{24}+\frac{1}{2} \sum_{n \neq 0} \frac{n q^{n^{2}}}{1-q^{n}} .
$$

\footnotetext{
${ }^{1}$ Ordinary mock theta functions of weight $k$ correspond to $k^{\prime}=0$.

${ }^{2}$ Our study of this type of functions, which are not, but, nearly holomorphic, may remind one of so-called BCOV equations, as remarked by Joyce to the authors (see [17, Section 6.3]).
} 
Here, $\sum_{n>0} n q^{n^{2}}$ is an example of half-theta functions (a.k.a. false-theta functions), which were studied by Rogers [29]. For half-period Jacobi theta functions $($ at $z=0) \theta_{1}(\tau)=\sum_{n \in \mathbb{Z}} \mathrm{e}^{\pi \mathrm{i} n^{2} \tau}$ and $\theta_{3}(\tau)=\sum_{n \in \mathbb{Z}} \mathrm{e}^{\pi \mathrm{i}\left(n+\frac{1}{2}\right)^{2} \tau}$, we have the following.

Theorem 1.2. For $\mathrm{SL}(2, \mathbb{Z})$, there is a unique real analytic modular form of weight two $\tilde{g}(\tau)$ with bounded growth at the cusp such that $\bar{D}(\tilde{g}(\tau))=$ $-\frac{\theta_{1}(2 \tau) \overline{\theta_{1}(2 \tau)}+\theta_{3}(2 \tau) \overline{\theta_{3}(2 \tau)}}{64 \pi^{2} y^{\frac{3}{2}}}$. The holomorphic part of $\tilde{g}(\tau)$ coincides with $\mathcal{J}_{2}(\tau)$.

Let us explain some properties of $\tilde{g}(\tau)$ in Theorem 1.2. The shadow of $\tilde{g}(\tau)$ is in the space $\frac{M^{\frac{1}{2}} \otimes \overline{M^{\frac{1}{2}}}}{y^{\frac{3}{2}}}$. The holomorphic part of $\tilde{g}(\tau)$ is a sum of products of classical theta functions of weight $\frac{1}{2}$ and ordinary mock theta functions of weight $\frac{3}{2}$, which will be derived from the Lerch function [35]. We are aware that we are leaving many questions open. For example, we would want some understanding of moduli stacks of cases other than ones considered here and $\mathcal{J}_{k}$ for $k \neq 2$, but it is our impression that they rather pose fundamental questions on stabilizer groups of objects, algebras associated to moduli-stack counting and mock theta functions. ${ }^{3}$

\section{Definitions}

\subsection{Stability conditions}

In this paper, our triangulated category $\mathcal{T}$ is assumed to be $\mathrm{D}(X)$ for some $\mathrm{K} 3$ surface $X$. For this $\mathcal{T}$, let us recall fundamental notions from [3].

For each object $E \in \mathcal{T}$, let us recall $[E]:=\operatorname{ch}(E) \sqrt{\operatorname{Td}(X)} \in H^{*}(X, \mathbb{Z})$ is the Mukai vector of $E$ and the lattice $H^{*}(X, \mathbb{Z})$ can be equipped with the Mukai paring, which was given in the introduction. We denote the lattice with the paring, called the Mukai lattice, as $N(\mathcal{T})$.

A stability condition $\sigma=(Z, \mathcal{P})$ for this $\mathcal{T}$ consists of a central charge $Z \in \operatorname{Hom}(N(\mathcal{T}), \mathbb{C})$ and a slicing $\mathcal{P}$, which is a family of full subcategories $\mathcal{P}(\phi)$ of $\mathcal{T}$ indexed by real numbers $\phi$ with the following conditions.

\footnotetext{
${ }^{3}$ After appearance of this paper, Zagier suggested a different way to construct $\tilde{g}(\tau)$, which suggests a way to show modularity properties of $\mathcal{J}_{k}$ for $k>2$. They appear to be more complicated than $\mathcal{J}_{2}$, but to understand them in terms of mock theta functions, one should also apply certain differential operators (the RankinCohen brackets). This will be studied in a separate publication.
} 
- If for some $\phi \in \mathbb{R}, E$ is a nonzero object in $\mathcal{P}(\phi)$, then for some positive real number $m(E)$, called the mass of $E, Z(E)=m(E) \exp (\mathrm{i} \pi \phi)$.

- For each real number $\phi, \mathcal{P}(\phi+1)=\mathcal{P}(\phi)[1]$.

- For real numbers $\phi_{1}>\phi_{2}$ and objects $A_{i} \in \mathcal{P}\left(\phi_{i}\right), \operatorname{Hom}_{\mathcal{T}}\left(A_{1}, A_{2}\right)=0$.

- For any nonzero object $E \in \mathcal{T}$, there exist real numbers $\phi_{1}>\cdots>$ $\phi_{n}$ and objects $A_{i} \in \mathcal{P}\left(\phi_{i}\right)$ such that there exists a sequence of exact triangles $E_{i-1} \rightarrow E_{i} \rightarrow A_{i}$ with $E_{0} \cong 0$ and $E_{n} \cong E$.

The sequence above is called the Harder-Narasimhan filtration (HNfiltration in short) of $E$. The HN-filtration of any nonzero object is unique up to isomorphisms. For each $\phi \in \mathbb{R}$, nonzero objects in $\mathcal{P}(\phi)$ are called semistable with phase $\phi$. If moreover a semistable object in $\mathcal{P}(\phi)$ has only the trivial Jordan-Hölder filtration in $\mathcal{P}(\phi)$, then it is called stable.

A stability condition is called numerically faithful [26, Definition 3.1] (faithful in short), if for each real number $r$, we have a primitive Mukai vector $v$ such that for each semistable object $E$ of the phase $r,[E]$ is a sum of $v$. Here, by [3, Proposition 8.3], $\operatorname{Stab}^{*}(X)$ satisfies the assumption of [26, Lemma 3.1], and so faithful stability conditions exist as a dense subset in $\operatorname{Stab}^{*}(X)$.

\subsection{Joyce invariants}

For each $r \in \mathbb{R}$ and Mukai vectors of semistable objects $E$ and $E^{\prime}$ with phase $r$, let us recall the algebra $C_{r}$ defined as $v_{[E]} * v_{\left[E^{\prime}\right]}=q^{-[E] \cdot\left[E^{\prime}\right]} v_{\left[E \oplus E^{\prime}\right]}[18$, Definition 6.3]; then, formally, we can put Joyce invariants $J^{v_{[E]}}(\sigma)$ as follows (see [15, Definition 6.22] and [31, Definition 5.9]):

$$
\sum_{[E] \in C_{r}} J^{v_{[E]}}(\sigma) v_{[E]}=\log \left(1+\sum_{[E] \in C_{r}} I\left(M^{v_{[E]}}(\sigma)\right) v_{[E]}\right)
$$

A few comments are in order. For certain moduli stacks indexed by multiples of a primitive Mukai vector, we see that by taking $I$ to be the extension of virtual Hodge numbers and taking $v_{[E]} * v_{\left[E^{\prime}\right]}=v_{\left[E \oplus E^{\prime}\right]}$ on multiples of a primitive Mukai vector, invariants in [24] are defined as above. On the other hand, quantum corrections $q^{-[E] \cdot\left[E^{\prime}\right]}$ give us quantum tori, which is an important topic in [20]. 


\subsection{Modular forms}

Let us recall the definition and properties of the Dedekind eta function (we denote $q=\mathrm{e}^{2 \pi \mathrm{i} \tau}$ ), where $\tau$ belongs to the upper half-plane.

$$
\eta(\tau)=\mathrm{e}^{\frac{\pi \mathrm{i} \tau}{12}} \prod_{n=1}^{\infty}\left(1-\mathrm{e}^{2 \pi \mathrm{i} n \tau}\right)=q^{1 / 24}\left(1-q-q^{2}+q^{5}+q^{7}+\cdots\right) .
$$

The eta function transforms like a modular form of weight $\frac{1}{2}$ :

$$
\eta(\tau+1)=\mathrm{e}^{\frac{\pi \mathrm{i}}{12}} \eta(\tau), \quad \eta\left(\frac{-1}{\tau}\right)=\mathrm{e}^{\frac{\pi \mathrm{i}}{4}} \sqrt{\tau} \eta(\tau)
$$

Next, we recall the half-period Jacobi theta functions (at $z=0$ ); note that we slightly changed the indexing:

$$
\begin{aligned}
& \theta_{1}(\tau)=\theta_{00}(0 ; \tau)=\sum_{n \in \mathbb{Z}} \mathrm{e}^{\pi \mathrm{i} n^{2} \tau} \quad=1+2 q^{\frac{1}{2}}+2 q^{2}+2 q^{\frac{9}{2}}+2 q^{8}+\cdots \\
& \theta_{2}(\tau)=\theta_{01}(0 ; \tau)=\sum_{n \in \mathbb{Z}}(-1)^{n} \mathrm{e}^{\pi \mathrm{i} n^{2} \tau}=1-2 q^{\frac{1}{2}}+2 q^{2}-2 q^{\frac{9}{2}}+2 q^{8}+\cdots \\
& \theta_{3}(\tau)=\theta_{10}(0 ; \tau)=\sum_{n \in \mathbb{Z}} \mathrm{e}^{\pi \mathrm{i}\left(n+\frac{1}{2}\right)^{2} \tau} \quad=2 q^{\frac{1}{8}}+2 q^{\frac{9}{8}}+2 q^{\frac{25}{8}}+2 q^{\frac{49}{8}}+\cdots
\end{aligned}
$$

We list their transformation properties as follows:

$$
\begin{array}{ll}
\theta_{1}(\tau+1)=\theta_{2}(\tau), & \theta_{1}\left(-\frac{1}{\tau}\right)=\mathrm{e}^{-\frac{\pi \mathrm{i}}{4}} \sqrt{\tau} \theta_{1}(\tau), \\
\theta_{2}(\tau+1)=\theta_{1}(\tau), & \theta_{2}\left(-\frac{1}{\tau}\right)=\mathrm{e}^{-\frac{\pi \mathrm{i}}{4}} \sqrt{\tau} \theta_{3}(\tau), \\
\theta_{3}(\tau+1)=\mathrm{e}^{\frac{\pi \mathrm{i}}{4}} \theta_{3}(\tau), & \theta_{3}\left(-\frac{1}{\tau}\right)=\mathrm{e}^{-\frac{\pi \mathrm{i}}{4}} \sqrt{\tau} \theta_{2}(\tau) .
\end{array}
$$

In particular, they are modular forms for the group $\Gamma(2)$.

We will also use the classical Eisenstein series of weight 2 for $\operatorname{SL}(2, \mathbb{Z})$ :

$$
E_{2}(\tau)=24 \frac{\eta^{\prime}(\tau)}{\eta(\tau)}=1-24 \sum_{k, n=1}^{\infty} k q^{n k}=1-24 q-72 q^{2}-96 q^{3}-\cdots
$$




\subsection{The Lerch function}

Having introduced some classical modular forms, we turn to the thesis of Zwegers [35]. In this thesis, we find the following definition of the Lerch function:

$$
\mu(u, v ; \tau)=\frac{\mathrm{e}^{\pi \mathrm{i} u}}{\theta(v ; \tau)} \sum_{n \in \mathbb{Z}} \frac{(-1)^{n} \mathrm{e}^{\pi \mathrm{i}\left(n^{2}+n\right) \tau+2 \pi \mathrm{i} n v}}{1-\mathrm{e}^{2 \pi \mathrm{i} n \tau+2 \pi \mathrm{i} u}} \quad(u, v \in \mathbb{C} \backslash(\mathbb{Z} \tau+\mathbb{Z}))
$$

The definition of the theta function he uses is the following one:

$$
\theta(z ; \tau)=\sum_{\nu \in \frac{1}{2}+\mathbb{Z}} \mathrm{e}^{\pi \mathrm{i} \nu^{2} \tau+2 \pi \mathrm{i} \nu\left(z+\frac{1}{2}\right)}
$$

The theta functions $\theta_{1}, \theta_{2}$ and $\theta_{3}$ are related to $\theta$ in the following way:

$$
\begin{aligned}
\theta\left(\frac{\tau}{2} ; \tau\right) & =-\mathrm{ie}^{-\frac{\pi \mathrm{i} \tau}{4}} \theta_{2}(\tau), \\
\theta\left(\frac{1+\tau}{2} ; \tau\right) & =-\mathrm{e}^{-\frac{\pi \mathrm{i} \tau}{4}} \theta_{1}(\tau), \\
\theta\left(\frac{1}{2} ; \tau\right) & =-\theta_{3}(\tau) .
\end{aligned}
$$

Zwegers found a way to add a correction term to $\mu$ so that the new function $\widetilde{\mu}$ has good transformation properties. Namely, he defines

$$
\widetilde{\mu}(u, v ; \tau)=\mu(u, v ; \tau)+\frac{\mathrm{i}}{2} R(u-v ; \tau)
$$

where

$$
\begin{aligned}
R(u ; \tau)= & \sum_{\nu \in \frac{1}{2}+\mathbb{Z}}\left\{\operatorname{sign}(\nu)-E\left(\left(\nu+\frac{\Im u}{y}\right) \sqrt{2 y}\right)\right\} \\
& \times(-1)^{\nu-\frac{1}{2}} \mathrm{e}^{-\pi \mathrm{i} \nu^{2} \tau-2 \pi \mathrm{i} \nu u} .
\end{aligned}
$$

Here $y=\Im \tau$ and $E$ is the function

$$
E(z)=2 \int_{0}^{z} \mathrm{e}^{-\pi t^{2}} d t=1-\operatorname{erfc}(z \sqrt{\pi})
$$


The result of Zwegers is the following transformation properties of $\widetilde{\mu}$ :

Theorem 2.1 [35, Theorem 1.11]. The function $\widetilde{\mu}$ satisfies

$$
\widetilde{\mu}(u, v ; \tau)=\widetilde{\mu}(v, u ; \tau)=\widetilde{\mu}(-u,-v ; \tau),
$$

and

$$
\begin{aligned}
\widetilde{\mu}(u, v ; \tau+1) & =\mathrm{e}^{-\frac{\pi \mathrm{i}}{4}} \widetilde{\mu}(u, v ; \tau), \\
\widetilde{\mu}\left(\frac{u}{\tau}, \frac{v}{\tau} ;-\frac{1}{\tau}\right) & =-\mathrm{e}^{-\frac{\pi \mathrm{i}}{4}-\frac{\pi \mathrm{i}(u-v)^{2}}{\tau}} \sqrt{\tau} \widetilde{\mu}(u, v ; \tau), \\
\widetilde{\mu}(u+1, v ; \tau) & =-\widetilde{\mu}(u, v ; \tau), \\
\widetilde{\mu}(u+\tau, v ; \tau) & =-\mathrm{e}^{2 \pi \mathrm{i}(u-v)+\pi \mathrm{i} \tau} \widetilde{\mu}(u, v) .
\end{aligned}
$$

Here is a list of properties that the functions $R$ and $\mu$ satisfy separately:

Proposition 2.1 [35, Propositions 1.4 and 1.9]. The functions $\mu$ and $R$ satisfy

$$
\mu(u, v ; \tau)=\mu(v, u ; \tau)=\mu(-u,-v ; \tau), \quad R(-z ; \tau)=R(z ; \tau),
$$

and we have

$$
\mu(u+1, v ; \tau)=-\mu(u, v ; \tau), \quad R(z+1 ; \tau)=-R(z ; \tau)
$$

We also mention one last property which we will use:

Proposition 2.2 [35, Proposition 1.4 and Theorem 1.11]. Both the functions $\mu$ and $\tilde{\mu}$ (if you plug it in place of $\mu$ ) satisfy

$$
\mu(u+z, v+z ; \tau)-\mu(u, v ; \tau)=\frac{\mathrm{i} \eta^{3}(\tau) \theta(u+v+z ; \tau) \theta(z ; \tau)}{\theta(u ; \tau) \theta(v ; \tau) \theta(u+z ; \tau) \theta(v+z ; \tau)}
$$

for $u, v, u+z, v+z \notin \mathbb{Z}+\tau \mathbb{Z}$.

\section{Proofs}

Let us prove Theorem 1.1.

Proof. For faithful stability conditions $\sigma$, in terms of Mukai vectors, $J^{\alpha}(\sigma)$ admit unique expressions. Since by [31, Theorem 1.5], we have $J^{\alpha}(\sigma)=$ $J^{\alpha}\left(\sigma^{\prime}\right)$ for any $\alpha$, especially for primitive ones, the statement follows. 
We will prove Corollary 1.1. Let us recall that an object $E \in \mathcal{T}$ is called spherical if $\operatorname{Ext}^{i}(E, E)=\mathbb{C}$ for $i=0,2$ and $\operatorname{Ext}^{i}(E, E)=0$ for else; spherical Mukai vectors are the ones of spherical objects.

Proof. By Theorem 1.1 and [26, Proposition 4.9], it is enough to show that for each spherical Mukai vector $\alpha$, we have a faithful stability condition with a semistable object of $\alpha$. For the case when $\alpha$ has a nonzero rank, by [32, Theorem 0.1(1)] and [3, Proposition 14.2], for some faithful $\sigma \in \operatorname{Stab}^{*}(X)$, we have a semistable spherical object of $\alpha$. For other cases, by [13, Chapter 5, Lemma 25], the first Chern class of $\alpha$ is either effective or anti-effective. So, by replacing $\alpha$ with $-\alpha$, if necessarily, one recalls that some coherent sheaf $E$ supported over a rational curve with $[E]=\alpha$ is Gieseker semistable. Then, by [31, Theorem 6.6], the statement follows.

A consequence of Corollary 1.1 is that, as pointed out to the authors by Bridgeland, the existence of a stable spherical object for each spherical Mukai vector gives another way to prove that $\operatorname{Stab}^{*}(X)$ is locally a bundle over the period domain of $X$, which consists of complexified Kähler classes of $X$ without ones that are orthogonal to spherical Mukai vectors. Let us prove Corollary 1.2.

Proof. Since $\alpha . \alpha=2$, by choosing a faithful $\sigma$, for positive integers $k_{i}$, we have

$$
J^{n \alpha}(\sigma)=\sum_{m=1}^{\infty} \sum_{k_{1}+\cdots+k_{m}=n} q^{\sum_{i>j} 2 k_{i} k_{j}} \frac{(-1)^{n-1}}{n} \Pi_{i=1}^{n} \frac{1}{I\left(\mathrm{GL}\left(k_{i}, \mathbb{C}\right)\right)} .
$$

Since $\sum_{i>j} 2 k_{i} k_{j}=\left(\sum k_{i}\right)^{2}-\sum k_{i}^{2}=n^{2}-\sum k_{i}^{2}$, we have

$$
J^{n \alpha}(\sigma)=q^{n^{2}} \sum_{m=1}^{\infty} \sum_{k_{1}+\cdots+k_{m}=n} \frac{(-1)^{n-1}}{n} \Pi_{i=1}^{n} \frac{q^{-k_{i}^{2}}}{I\left(\mathrm{GL}\left(k_{i}, \mathbb{C}\right)\right)} .
$$

Let $\quad F(x)=\sum_{m \geq 0} \frac{q^{-m^{2}}}{I(\mathrm{GL}(m, \mathbb{C}))} x^{m}$. Then $F(x)-F(q x)=x F(x)$ and $J^{n \alpha}(\sigma)$ is the $n$th coefficient of $q^{n^{2}} \sum \frac{(-1)^{n-1}}{n}(F(x)-1)=q^{n^{2}} \log F(x)$. Since $\log F(x)+\log (1-x)=\log F(q x)$, the $n$th coefficient of $\log F(x)$ is $\frac{1}{n\left(1-q^{n}\right)}$. So the statement follows.

The rest of this section is devoted to the proof of Theorem 1.2. We need to construct a function with good transformation properties whose holomorphic part is $\mathcal{J}_{2}$. We are going to use $\widetilde{\mu}$ at "points of order two." The values at these points are not interesting because one can check that 


\section{Proposition 3.1.}

$$
\widetilde{\mu}\left(\frac{1}{2}, \frac{\tau}{2} ; \tau\right)=\widetilde{\mu}\left(\frac{1}{2}, \frac{1+\tau}{2} ; \tau\right)=\widetilde{\mu}\left(\frac{\tau}{2}, \frac{1+\tau}{2} ; \tau\right)=0
$$

Therefore, we should look at the derivatives of $\widetilde{\mu}$ at the points of order 2 . Namely, we define

$$
\begin{aligned}
& \mu_{1}(\tau)=\left.\frac{d}{2 \pi \mathrm{i} d s}\right|_{s=0} \widetilde{\mu}\left(\frac{1}{2}, \frac{\tau}{2}+s ; \tau\right), \\
& \mu_{2}(\tau)=\left.\frac{d}{2 \pi \mathrm{i} d s}\right|_{s=0} \widetilde{\mu}\left(\frac{1}{2}, \frac{1+\tau}{2}+s ; \tau\right), \\
& \mu_{3}(\tau)=\left.\frac{d}{2 \pi \mathrm{i} d s}\right|_{s=0} \widetilde{\mu}\left(\frac{\tau}{2}, \frac{1+\tau}{2}+s ; \tau\right) .
\end{aligned}
$$

Applying Theorem 2.1, we obtain the transformation properties of $\mu_{k}$.

Proposition 3.2. We have

$$
\begin{aligned}
& \mu_{1}(1+\tau)=\mathrm{e}^{-\frac{\pi \mathrm{i}}{4}} \mu_{2}(\tau), \quad \mu_{2}(1+\tau)=-\mathrm{e}^{-\frac{\pi \mathrm{i}}{4}} \mu_{1}(\tau), \\
& \mu_{3}(1+\tau)=\mathrm{e}^{-\frac{\pi \mathrm{i}}{4}}\left(\frac{\theta_{3}(\tau)^{3}}{4}+\mu_{3}(\tau)\right), \\
& \mu_{1}\left(-\frac{1}{\tau}\right)=\mathrm{e}^{\frac{\pi \mathrm{i}}{4}-\pi \mathrm{i} \frac{1+\tau^{2}}{4 \tau} \tau^{\frac{3}{2}}}\left(\frac{\mathrm{e}^{\frac{\pi \mathrm{i} \tau}{4}} \theta_{1}(\tau)^{3}}{4}+\mu_{1}(\tau)\right), \\
& \mu_{2}\left(-\frac{1}{\tau}\right)=\mathrm{e}^{-\frac{\pi \mathrm{i}}{4}-\frac{\pi \mathrm{i}}{4 \tau}} \tau^{\frac{3}{2}} \mu_{3}(\tau), \quad \mu_{3}\left(-\frac{1}{\tau}\right)=-\mathrm{e}^{-\frac{\pi \mathrm{i}}{4}-\frac{\pi \mathrm{i} \tau}{4}} \tau^{\frac{3}{2}} \mu_{2}(\tau) .
\end{aligned}
$$

Thus, we modify the functions $\mu_{k}$ in order to obtain better transformation properties as follows:

$$
\begin{aligned}
& \widetilde{h}_{1}(\tau)=\mathrm{e}^{-\frac{\pi \mathrm{i} \tau}{4}} \mu_{1}(\tau)+\frac{\theta_{1}(\tau)^{3}}{8}, \\
& \widetilde{h}_{2}(\tau)=-\mathrm{ie}^{-\frac{\pi \mathrm{i} \tau}{4}} \mu_{2}(\tau)+\frac{\theta_{2}(\tau)^{3}}{8}, \\
& \widetilde{h}_{3}(\tau)=-\mu_{3}(\tau)-\frac{\theta_{3}(\tau)^{3}}{8} .
\end{aligned}
$$


Then, we have

$$
\begin{array}{ll}
\widetilde{h}_{1}(\tau+1)=\widetilde{h}_{2}(\tau), & \widetilde{h}_{1}\left(-\frac{1}{\tau}\right)=\mathrm{e}^{\frac{\pi \mathrm{i}}{4}} \tau^{\frac{3}{2}} \widetilde{h}_{1}(\tau), \\
\widetilde{h}_{2}(\tau+1)=\widetilde{h}_{1}(\tau), & \widetilde{h}_{2}\left(-\frac{1}{\tau}\right)=\mathrm{e}^{\frac{\pi \mathrm{i}}{4}} \tau^{\frac{3}{2}} \widetilde{h}_{3}(\tau), \\
\widetilde{h}_{3}(\tau+1)=\mathrm{e}^{-\frac{\pi \mathrm{i}}{4}} \widetilde{h}_{3}(\tau), & \widetilde{h}_{3}\left(-\frac{1}{\tau}\right)=\mathrm{e}^{\frac{\pi \mathrm{i}}{4}} \tau^{\frac{3}{2}} \widetilde{h}_{2}(\tau) .
\end{array}
$$

Now, we need to compute the decomposition $\widetilde{h}_{k}=h_{k}+R_{k}$ coming from the corresponding decomposition (2.1) of $\widetilde{\mu}$. Differentiating $R$, we obtain

\section{Proposition 3.3.}

$$
\begin{aligned}
\left.\frac{d}{2 \pi \mathrm{i} d s}\right|_{s=0} R\left(\frac{1-\tau}{2}-s ; \tau\right)= & -\mathrm{i} \mathrm{e}^{\frac{\pi \mathrm{i} \tau}{4}}\left(\sum_{n \in \mathbb{Z}}|n| \beta\left(2 y n^{2}\right) \mathrm{e}^{-\pi \mathrm{i} n^{2} \tau}\right. \\
& \left.+\frac{1}{2}-\frac{\overline{\theta_{1}(\tau)}}{\pi \sqrt{2 y}}\right), \\
\left.\frac{d}{2 \pi \mathrm{i} d s}\right|_{s=0} R\left(-\frac{\tau}{2}-s ; \tau\right)= & \mathrm{e}^{\frac{\pi \mathrm{i} \tau}{4}}\left(\sum_{n \in \mathbb{Z}}(-1)^{n}|n| \beta\left(2 y n^{2}\right) \mathrm{e}^{-\pi \mathrm{i} n^{2} \tau}\right. \\
& \left.+\frac{1}{2}-\frac{\theta_{2}(\tau)}{\pi \sqrt{2 y}}\right), \\
\left.\frac{d}{2 \pi \mathrm{i} d s}\right|_{s=0} R\left(-\frac{1}{2}-s ; \tau\right)= & \mathrm{i}\left(\sum_{\nu \in \mathbb{Z}+\frac{1}{2}}|\nu| \beta\left(2 y \nu^{2}\right) \mathrm{e}^{-\pi \mathrm{i} \nu^{2} \tau}-\frac{\overline{\theta_{3}(\tau)}}{\pi \sqrt{2 y}}\right) .
\end{aligned}
$$

Here we use $\beta$ instead of $E$,

$$
\beta(x)=\int_{x}^{\infty} t^{-\frac{1}{2}} \mathrm{e}^{-\pi t} d t=1-E(\sqrt{x})=\operatorname{erfc}(\sqrt{\pi x})
$$

It remains to differentiate the function $\mu$. 
Proposition 3.4. The corresponding derivatives of $\mu$ are given by

$$
\begin{aligned}
\left.\frac{d}{2 \pi \mathrm{i} d s}\right|_{s=0} \mu & \left(\frac{1}{2}, \frac{\tau}{2}+s ; \tau\right) \\
= & -\frac{\mathrm{e}^{\frac{\pi \mathrm{i} \tau}{4}}}{24 \theta_{1}(\tau)}\left(-2+6 \theta_{1}(\tau)+3 \theta_{1}^{4}(\tau)-E_{2}(\tau)\right. \\
& \left.+48 \sum_{n=1}^{\infty} \frac{\mathrm{e}^{\pi \mathrm{i}\left(n^{2}+2 n\right) \tau}}{\left(1-\mathrm{e}^{2 \pi \mathrm{i} n \tau}\right)^{2}}\right), \\
\left.\frac{d}{2 \pi \mathrm{i} d s}\right|_{s=0} \mu\left(\frac{1}{2}, \frac{1+\tau}{2}+s ; \tau\right) & -\mathrm{i} \frac{\mathrm{e}^{\frac{\pi \mathrm{i} \tau}{4}}}{24 \theta_{2}(\tau)}\left(-2+6 \theta_{2}(\tau)+3 \theta_{2}^{4}(\tau)-E_{2}(\tau)\right. \\
& \left.+48 \sum_{n=1}^{\infty} \frac{(-1)^{n} \mathrm{e}^{\pi \mathrm{i}\left(n^{2}+2 n\right) \tau}}{\left(1-\mathrm{e}^{2 \pi \mathrm{i} n \tau}\right)^{2}}\right), \\
\left.\frac{\mu}{2 \pi \mathrm{i} d s}\right|_{s=0} & \left(\frac{\tau}{2}, \frac{1+\tau}{2}+s ; \tau\right) \\
= & \frac{1}{24 \theta_{3}(\tau)}\left(1-3 \theta_{3}(\tau)^{4}-E_{2}(\tau)\right. \\
& \left.+24 \sum_{n=1}^{\infty} \mathrm{e}^{\pi \mathrm{i}\left(n^{2}+n\right) \tau} \frac{1+\mathrm{e}^{2 \pi \mathrm{i} n \tau}}{\left(1-\mathrm{e}^{2 \pi \mathrm{i} n \tau}\right)^{2}}\right) .
\end{aligned}
$$

Proof. In this case the computations are more complicated, so we give a proof of the first identity. The remaining identities are proved in a similar way. We use the following decomposition of $\mu$ :

$$
\begin{aligned}
\mu(s, z ; \tau)= & \frac{\mathrm{e}^{\pi \mathrm{i} s}}{\theta(z ; \tau)\left(1-\mathrm{e}^{2 \pi \mathrm{i} s}\right)} \\
& +\frac{1}{\theta(z ; \tau)} \sum_{n=1}^{\infty}(-1)^{n} \mathrm{e}^{\pi \mathrm{i}\left(n^{2}+n\right) \tau} \\
& \times\left(\frac{\mathrm{e}^{2 \pi \mathrm{i} n z+\pi \mathrm{i} s}}{1-\mathrm{e}^{2 \pi \mathrm{i} n \tau+2 \pi \mathrm{i} s}}-\frac{\mathrm{e}^{-2 \pi \mathrm{i} n z-\pi \mathrm{i} s}}{1-\mathrm{e}^{2 \pi \mathrm{i} n \tau-2 \pi \mathrm{i} s}}\right) .
\end{aligned}
$$

We compute the Taylor expansion with respect to $2 \pi \mathrm{i} s$ around $s=0$ of the expression above for $z=\frac{1+\tau}{2}$. We need only the coefficient at $2 \pi \mathrm{i} s$. This 
coefficient equals

$$
\begin{aligned}
-\frac{\mathrm{e}^{\frac{\pi \mathrm{i} \tau}{4}}}{\theta_{1}(\tau)}\left(\frac{1}{24}+\sum_{n=1}^{\infty} \mathrm{e}^{\pi \mathrm{i} n^{2} \tau} \frac{\left(1+\mathrm{e}^{2 \pi \mathrm{i} n \tau}\right)^{2}}{2\left(1-\mathrm{e}^{2 \pi \mathrm{i} n \tau}\right)^{2}}\right) \\
=-\frac{\mathrm{e}^{\frac{\pi \mathrm{i} \tau}{4}}}{\theta_{1}(\tau)}\left(-\frac{5}{24}+\frac{\theta_{1}(\tau)}{4}+2 \sum_{n=1}^{\infty} \frac{\mathrm{e}^{\pi \mathrm{i}\left(n^{2}+2 n\right) \tau}}{\left(1-\mathrm{e}^{2 \pi \mathrm{i} n \tau}\right)^{2}}\right)
\end{aligned}
$$

In order to apply Proposition 2.2, we also need to compute the coefficient at $2 \pi \mathrm{i} s$ of the following expression (we omit $\tau$ from the arguments of $\theta$ ):

$$
\frac{\mathrm{i} \eta(\tau)^{3} \theta\left(\frac{1}{2}+s\right) \theta\left(\frac{\tau}{2}\right)}{\theta\left(\frac{1-\tau}{2}\right) \theta\left(\frac{1}{2}\right) \theta(s) \theta\left(\frac{\tau}{2}+s\right)} .
$$

For this we need to compute the Taylor expansions of $\theta(s), \theta\left(\frac{1}{2}+s\right)$ and $\theta\left(\frac{\tau}{2}+s\right)$ up to the second term with respect to $2 \pi \mathrm{i} s$. We have (denoting by ' the operator $\left.\frac{d}{2 \pi \mathrm{i} d \tau}\right)$ :

$$
\begin{gathered}
\theta(s)=(2 \pi \mathrm{i} s) \mathrm{i} \eta(\tau)^{3}\left(1+(2 \pi \mathrm{i} s)^{2} \frac{\eta^{\prime}(\tau)}{\eta(\tau)}\right)+\cdots \\
\theta\left(\frac{1}{2}+s\right)=-\theta_{3}(\tau)\left(1+(2 \pi \mathrm{i} s)^{2} \frac{\theta_{3}^{\prime}(\tau)}{\theta_{3}(\tau)}\right)+\cdots, \\
\theta\left(\frac{\tau}{2}+s\right)=-\mathrm{ie}^{-\frac{\pi \mathrm{i} \tau}{4}} \theta_{2}(\tau)\left(1-\frac{2 \pi \mathrm{i} s}{2}+(2 \pi \mathrm{i} s)^{2}\left(\frac{1}{8}+\frac{\theta_{2}^{\prime}(\tau)}{\theta_{2}(\tau)}\right)\right)+\cdots .
\end{gathered}
$$

Thus, the coefficient at $2 \pi \mathrm{i} s$ of the expression in question is given by

$$
-\frac{\mathrm{e}^{\frac{\pi \mathrm{i} \tau}{4}}}{\theta_{1}(\tau)}\left(\frac{\theta_{3}^{\prime}(\tau)}{\theta_{3}(\tau)}-\frac{\eta^{\prime}(\tau)}{\eta(\tau)}+\frac{1}{8}-\frac{\theta_{2}^{\prime}(\tau)}{\theta_{2}(\tau)}\right) .
$$

Putting everything together

$$
\begin{aligned}
& \left.\frac{d}{2 \pi \mathrm{i} d s}\right|_{s=0} \mu\left(\frac{1}{2}, \frac{\tau}{2}+s ; \tau\right) \\
& =\left.\frac{d}{2 \pi \mathrm{i} d s}\right|_{s=0}\left(\mu\left(\frac{1-\tau}{2}, s ; \tau\right)+\frac{\mathrm{i} \eta(\tau)^{3} \theta\left(\frac{1}{2}+s\right) \theta\left(\frac{\tau}{2}\right)}{\theta\left(\frac{1-\tau}{2}\right) \theta\left(\frac{1}{2}\right) \theta(s) \theta\left(\frac{\tau}{2}+s\right)}\right) \\
& =-\frac{\mathrm{e}^{\frac{\pi \mathrm{i} \tau}{4}}}{\theta_{1}(\tau)}\left(-\frac{1}{12}+\frac{\theta_{1}(\tau)}{4}+\frac{\theta_{3}^{\prime}(\tau)}{\theta_{3}(\tau)}-\frac{\eta^{\prime}(\tau)}{\eta(\tau)}-\frac{\theta_{2}^{\prime}(\tau)}{\theta_{2}(\tau)}\right. \\
& \left.\quad+2 \sum_{n=1}^{\infty} \frac{\mathrm{e}^{\pi \mathrm{i}\left(n^{2}+2 n\right) \tau}}{\left(1-\mathrm{e}^{2 \pi \mathrm{i} n \tau}\right)^{2}}\right),
\end{aligned}
$$


and the following identity completes the proof

$$
\frac{\theta_{3}^{\prime}(\tau)}{\theta_{3}(\tau)}-\frac{\eta^{\prime}(\tau)}{\eta(\tau)}-\frac{\theta_{2}^{\prime}(\tau)}{\theta_{2}(\tau)}=\frac{\theta_{1}^{4}(\tau)}{8}-\frac{E_{2}(\tau)}{24}
$$

Propositions 3.3 and 3.4 together give the Fourier expansions of $\widetilde{h}_{k}$. Denote

$$
\begin{aligned}
& h_{1}(\tau)=\frac{1}{24 \theta_{1}(\tau)}\left(2+E_{2}(\tau)-48 \sum_{n=1}^{\infty} \frac{\mathrm{e}^{\pi \mathrm{i}\left(n^{2}+2 n\right) \tau}}{\left(1-\mathrm{e}^{2 \pi \mathrm{i} n \tau}\right)^{2}}\right), \\
& h_{2}(\tau)=\frac{1}{24 \theta_{2}(\tau)}\left(2+E_{2}(\tau)-48 \sum_{n=1}^{\infty} \frac{(-1)^{n} \mathrm{e}^{\pi \mathrm{i}\left(n^{2}+2 n\right) \tau}}{\left(1-\mathrm{e}^{2 \pi \mathrm{i} n \tau}\right)^{2}}\right), \\
& h_{3}(\tau)=\frac{1}{24 \theta_{3}(\tau)}\left(-1+E_{2}(\tau)-24 \sum_{n=1}^{\infty} \mathrm{e}^{\pi \mathrm{i}\left(n^{2}+n\right) \tau} \frac{1+\mathrm{e}^{2 \pi \mathrm{i} n \tau}}{\left(1-\mathrm{e}^{2 \pi \mathrm{i} n \tau}\right)^{2}}\right) .
\end{aligned}
$$

The series $h_{k}$ are holomorphic power series converging on the upper halfplane. Denote

$$
\begin{aligned}
& R_{1}(\tau)=\frac{1}{2} \sum_{n \in \mathbb{Z}}|n| \beta\left(2 y n^{2}\right) \mathrm{e}^{-\pi \mathrm{i} n^{2} \tau}-\frac{\overline{\theta_{1}(\tau)}}{2 \pi \sqrt{2 y}} \\
& R_{2}(\tau)=\frac{1}{2} \sum_{n \in \mathbb{Z}}(-1)^{n}|n| \beta\left(2 y n^{2}\right) \mathrm{e}^{-\pi \mathrm{i} n^{2} \tau}-\frac{\overline{\theta_{2}(\tau)}}{2 \pi \sqrt{2 y}} \\
& R_{3}(\tau)=\frac{1}{2} \sum_{\nu \in \mathbb{Z}+\frac{1}{2}}|\nu| \beta\left(2 y \nu^{2}\right) \mathrm{e}^{-\pi \mathrm{i} \nu^{2} \tau}-\frac{\overline{\theta_{3}(\tau)}}{2 \pi \sqrt{2 y}}
\end{aligned}
$$

Proposition 3.5. For $k=1,2,3$, we have

$$
\widetilde{h}_{k}(\tau)=h_{k}(\tau)+R_{k}(\tau)
$$

In his thesis Zwegers also represents $R$ as a certain integral involving a theta function of weight $\frac{3}{2}$. In a similar way, we obtain such a representation for $R_{k}$, but with theta functions of weight $\frac{1}{2}$.

Proposition 3.6. For $k=1,2,3$

$$
R_{k}(\tau)=\frac{1}{4 \pi \mathrm{i}} \overline{\int_{\tau}^{\mathrm{i} \infty} \frac{\theta_{k}(z) d z}{(-\mathrm{i}(z-\bar{\tau}))^{\frac{3}{2}}}} .
$$

We have slightly nicer expressions for the sums in the definitions of $h_{k}$. 


\section{Proposition 3.7.}

$$
\begin{aligned}
& 2 \sum_{n=1}^{\infty} \frac{\mathrm{e}^{\pi \mathrm{i}\left(n^{2}+2 n\right) \tau}}{\left(1-\mathrm{e}^{2 \pi \mathrm{i} n \tau}\right)^{2}}=\sum_{\substack{m>n>0, m-n \text { even }}} m \mathrm{e}^{\pi \mathrm{i} m n \tau}-\sum_{\substack{n>m>0, m-n \text { even }}} m \mathrm{e}^{\pi \mathrm{i} m n \tau}, \\
& 2 \sum_{n=1}^{\infty} \frac{(-1)^{n} \mathrm{e}^{\pi \mathrm{i}\left(n^{2}+2 n\right) \tau}}{\left(1-\mathrm{e}^{2 \pi \mathrm{i} n \tau}\right)^{2}}=\sum_{\substack{m>n>0, m-n \text { even }}} m(-1)^{m} \mathrm{e}^{\pi \mathrm{i} m n \tau} \\
& -\sum_{\substack{n>m>0 \\
m-n \text { even }}} m(-1)^{m} \mathrm{e}^{\pi \mathrm{i} m n \tau} \\
& \sum_{n=1}^{\infty} \mathrm{e}^{\pi \mathrm{i}\left(n^{2}+n\right) \tau} \frac{1+\mathrm{e}^{2 \pi \mathrm{i} n \tau}}{\left(1-\mathrm{e}^{2 \pi \mathrm{i} n \tau}\right)^{2}}=\sum_{\substack{m>n>0, m-n \text { odd }}} m \mathrm{e}^{\pi \mathrm{i} m n \tau}-\sum_{\substack{n>m>0, m-n \text { odd }}} m \mathrm{e}^{\pi \mathrm{i} m n \tau} .
\end{aligned}
$$

Now it is not hard to observe the following fact.

\section{Proposition 3.8. We have}

$$
h_{1}(\tau) \theta_{1}(\tau)+h_{2}(\tau) \theta_{2}(\tau)-4\left(h_{1}(4 \tau) \theta_{1}(4 \tau)+h_{3}(4 \tau) \theta_{3}(4 \tau)\right)=-\frac{\theta_{1}(2 \tau)^{4}}{4} .
$$

Using the corresponding identities between the theta functions, namely

$$
\theta_{1}(\tau)+\theta_{2}(\tau)=2 \theta_{1}(4 \tau), \quad \theta_{1}(\tau)+\theta_{3}(\tau)=\theta_{1}\left(\frac{\tau}{4}\right)
$$

the integral representation of $R_{k}$ from Proposition 3.6 and the change of variables

$$
\overline{4 \pi \mathrm{i} R_{k}(4 \tau)}=\int_{4 \tau}^{\mathrm{i} \infty} \frac{\theta_{k}(z) d z}{(-\mathrm{i}(z-4 \bar{\tau}))^{\frac{3}{2}}}=\frac{1}{2} \int_{\tau}^{\mathrm{i} \infty} \frac{\theta_{k}(4 z) d z}{(-\mathrm{i}(z-\bar{\tau}))^{\frac{3}{2}}}
$$

we obtain

$$
R_{1}(\tau) \theta_{1}(\tau)+R_{2}(\tau) \theta_{2}(\tau)-4\left(R_{1}(4 \tau) \theta_{1}(4 \tau)+R_{3}(4 \tau) \theta_{3}(4 \tau)\right)=0 .
$$

Thus, we have

\section{Proposition 3.9.}

$$
\widetilde{h}_{1}(\tau) \theta_{1}(\tau)+\widetilde{h}_{2}(\tau) \theta_{2}(\tau)-4\left(\widetilde{h}_{1}(4 \tau) \theta_{1}(4 \tau)+\widetilde{h}_{3}(4 \tau) \theta_{3}(4 \tau)\right)=-\frac{\theta_{1}(2 \tau)^{4}}{4}
$$


Therefore, we take

$$
\begin{aligned}
& \widetilde{g}(\tau)=-\frac{\widetilde{h}_{1}(2 \tau) \theta_{1}(2 \tau)+\widetilde{h}_{3}(2 \tau) \theta_{3}(2 \tau)}{2}+\frac{\theta_{1}(\tau)^{4}+\theta_{2}(\tau)^{4}}{96} \\
& g(\tau)=-\frac{h_{1}(2 \tau) \theta_{1}(2 \tau)+h_{3}(2 \tau) \theta_{3}(2 \tau)}{2}+\frac{\theta_{1}(\tau)^{4}+\theta_{2}(\tau)^{4}}{96}
\end{aligned}
$$

and

$$
r(\tau)=-\frac{R_{1}(2 \tau) \theta_{1}(2 \tau)+R_{3}(2 \tau) \theta_{3}(2 \tau)}{2}
$$

It is clear that

$$
\widetilde{g}(\tau)=g(\tau)+r(\tau)
$$

Proposition 3.9 and the transformation properties of $\widetilde{h}_{k}$ imply the following.

Proposition 3.10. The function $\widetilde{g}(\tau)$ transforms like a modular form of weight 2 , i.e.,

$$
\widetilde{g}(\tau+1)=\widetilde{g}(\tau), \quad \widetilde{g}\left(-\frac{1}{\tau}\right)=\tau^{2} \widetilde{g}(\tau)
$$

Using manipulations with power series as in Proposition 3.7 and the identity $4 E_{2}(2 \tau)-2 E_{2}(\tau)=\theta_{1}^{4}(\tau)+\theta_{2}(\tau)^{4}$, we obtain the Fourier expansion of $g$.

Proposition 3.11. We have

$$
g(\tau)=-\frac{E_{2}(\tau)}{24}-\frac{1}{2} \sum_{n \in \mathbb{Z} \backslash\{0\}} \frac{n q^{n^{2}}}{1-q^{n}}=-\frac{1}{24}+\sum_{n=1}^{\infty} \sigma^{\prime}(n) q^{n}
$$

where $\sigma^{\prime}(n)$ denotes the sum of positive divisors of $n$, which are greater than $\sqrt{n}$, plus half $\sqrt{n}$ in the case if $n$ is a perfect square.

Now, we complete our proof of Theorem 1.2. By Proposition 3.6, $r(\tau)=$ $R(\bar{D}(r(\tau)))=R(\bar{D}(\tilde{g}(\tau)))$, where $R$ is the operator from the introduction, hence $g$ is the holomorphic part of $\tilde{g}$. Proposition 3.11 gives the Fourier expansion of $g$, which, as one can easily verify, coincides with $\mathcal{J}_{2}$. Having the transformation properties of $\tilde{g}$ proved in Proposition 3.10, it remains to check only the uniqueness statement. This is obvious since there are no holomorphic modular forms of weight 2 for $\operatorname{SL}(2, \mathbb{Z})$. 


\section{Acknowledgments}

A.M. and S.O. thank Max-Planck-Institut für Mathematik and Institut des Hautes Études Scientifiques. They thank Aspinwall, Bridgeland, Bringmann, Ceyhan, Diaconescu, Dyson, Harder, Kajiura, Kontsevich, Mirković, Mizuno, Nakajima, Takahashi, Takagi, Toda, Yoshioka, Zagier and Zudilin for their helpful comments or discussions. Especially, Kontsevich and Zagier gave them their preprints [20] and [33] and made useful discussions and remarks. Bringmann read the preliminary version of this paper and made some corrections. S.O. thanks Australian National University and Research Institute of Mathematical Sciences for their support, University of Texas Austin for its support of his visit and active sessions with Ben-Zvi, Freed, Keel and Soibelman in November 2007 and Institute of Advanced Study for its support of his visit in March 2008; in particular, he thanks Dyson for his comment "Thank you very much. I am very glad to know it". S.O. is supported by JSPS Grant-in-aid 21840030 and GCOE Program at Kyoto University.

\section{References}

[1] D. Arcara, A. Bertram and M. Lieblich, Bridgeland-stable moduli spaces for K-trivial surfaces, with an appendix by M. Lieblich, arXiv:0708. 2247.

[2] T. Bridgeland and V. Toledano-Laredo, Stability conditions and Stoke's phenomena, arXiv:0801.3974.

[3] T. Bridgeland, Stability conditions on K3 surfaces, Duke Math. J. 141(2) (2008), 241-291, doi:10.1215/S0012-7094-08-14122-5, arXiv:math/0307164.

[4] T. Bridgeland, Stability conditions on triangulated categories, Ann. of Math. 166(2), (2007), 317-346, arXiv:math/0212237.

[5] T. Bridgeland, Spaces of stability conditions, Algebraic geometrySeattle 2005. Part 1, 1-21, Proceedings of Symposia in Pure Mathematics, 80, Part 1, American Mathematical Society, Providence, RI, 2009, arXiv:math.AG/0611510.

[6] K. Bringmann and K. Ono, Dyson's ranks and Maass forms, Ann. of Math. (2), to appear. 
[7] K. Bringmann and K. Ono, Lifting cusp forms to Maass forms with an application to partitions, Proc. Natl. Acad. Sci. USA 104(10) (2007), 3725-3731, doi:10.1073/pnas.0611414104.

[8] K. Bringmann and K. Ono, The $f(q)$ mock theta function conjecture and partition ranks, Invent. Math. 165(2) (2006), 243-266, doi:10.1007/s00222-005-0493-5.

[9] J. H. Bruinier and K. Ono, Heegner divisors, L-functions and harmonic weak Maass forms, Ann. of Math. (2), to appear, arXiv:0710.0283.

[10] Séminaire C. Chevalley; 2e année: 1958. Anneaux de Chow et application, Secrétariat mathématique, 11 rue Pierre Curie, Paris, 1958, pp. iii+134.

[11] M. R. Douglas, D-branes on Calabi-Yau manifolds, in European Congress of Mathematics, II (Barcelona, 2000), 449-466, Progr. Math. 202, Birkhäuser, Basel, 2001, arXiv:math/0009209.

[12] F. Dyson, A walk through Ramanujan's garden, Lecture given at the Ramanujan Centenary Conference (Illinois, 1987), in Selected Papers of Freeman Dyson with Commentary, Americal Mathematical Society, 1996, 187-208.

[13] R. Friedman, Algebraic surfaces and holomorphic vector bundles. Universitext. Springer-Verlag, New York, 1998. pp. $\mathrm{x}+328$.

[14] G. Harder and M. S. Narasimhan, On the cohomology groups of moduli spaces of vector bundles on curves. Math. Ann. 212 (1974/75), 215-248, doi:10.1007/BFraff+01357141.

[15] D. Joyce, Configurations in abelian categories. IV. Invariants and changing stability conditions, Adv. Math. 217(1) (2008), 125-204, doi:10.1016/j.aim.2007.06.011, arXiv:math/0410268.

[16] D. Joyce, Motivic invariants of Artin stacks and "stack functions", Quartery J Math., 58(3) (2007), 345-392, doi:10.1093/qmath/ham019, arXiv:math/0509722.

[17] D. Joyce, Holomorphic generating functions for invariants counting coherent sheaves on Calabi-Yau 3-folds, Geom. Topol. 11 (2007), 667725, doi:10.2140/gt.2007.11.667, arXiv: hep-th/0607039.

[18] D. Joyce, Configurations in abelian categories. II. Ringel-Hall algebras, Adv. Math. 210(2) (2007), 635-706, doi:10.1016/j.aim.2006.07.006, arXiv:math/0503029. 
[19] S. Katz and C.-C. Liu, Enumerative geometry of stable maps with Lagrangian boundary conditions and multiple covers of the disc, Adv. Theor. Math. Phys. 5(1) (2001), 1-49.

[20] M. Kontsevich and Y. Soibelman, Stability structures, motivic Donaldson-Thomas invariants and cluster transformations, arXiv:0811.2435.

[21] M. Kontsevich, Homological algebra of mirror symmetry, Proceedings of the International Congress of Mathematicians, 1, 2 (Zürich, 1994), Birkhäuser, Basel, 1995, 120-139.

[22] A. Kresch, Cycle groups for Artin stacks. Invent. Math. 138(3) (1999), 495-536, doi: 10.1007/s002220050351.

[23] S. Mukai, On the moduli space of bundles on K3 surfaces, I, Vector bundles on algebraic varieties (Bombay, 1984), Tata Institute of Fundamental Research Studies in Mathematics, 11, Tata Institute of Fundamental Research, Bombay, 1987, 341-413.

[24] H. Nakajima and K. Yoshioka, Instanton counting and Donaldson invariants (Japanese), Sūgaku 59(2) (2007), 131-153.

[25] S. Okada, Talk at AMS Summer Research Conference, Derived Categories in Mathematics and Physics, Snowbird, Utah, July 2007.

[26] S. Okada, Topologies on a triangulated category, arXiv:math/0701507.

[27] S. Ramanujan, Collected papers of Srinivasa Ramanujan (eds. G. H. Hardy, P. V. S. Aiyar, and B.M. Wilson), American Mathematical Soceity Providence, RI, 2000.

[28] S. Ramanujan, The lost notebook and other unpublished papers, Springer-Verlag, Berlin; Narosa Publishing House, New Delhi, 1988.

[29] L. J. Rogers, On two theorems of combinatory analysis and some allied identities, Lond. M. S. Proc. 16(2) (1917), 315-336.

[30] P. Seidel and R. Thomas, Braid group actions on derived categories of coherent sheaves, Duke Math. J. 108(1) (2001) 37-108, doi:10.1215/S0012-7094-01-10812-0, arXiv:math/0001043.

[31] Y. Toda, Moduli stacks and invariants of semistable objects on K3 surfaces, Adv. Math. 217(6) (2008) 2736-2781, doi:10.1016/j.aim.2007.11.010, arXiv:math/0703590. 
[32] K. Yoshioka, Irreducibility of moduli spaces of vector bundles on K3 surfaces, arXiv:math/9907001.

[33] D. Zagier, Ramanujan's mock theta functions and their applications, Séminaire Bourbaki, 60éme année, 2006-2007, 986.

[34] D. Zagier, The dilogarhithm functions, Frontiers in number theory, physics, and geometry, II, Springer, Berlin, 2007, 3-65.

[35] S. P. Zwegers, Mock theta functions, Utrecht $\mathrm{PhD}$ thesis, 2002, http://igitur-archive.library.uu.nl/dissertations/2003-0127-094324/ inhoud.htm.

MaX-Plank-Institut Für Mathematik (MPIM)

VIVATSGASSE 7

BONN D-53111

GERMANY

E-mail address: mellit@mpim-bonn.mpg.de

Research Institute for Mathematical Sciences (RIMS)

KYOTO UNIVERSITY

КҮОто 606-8502

JAPAN

E-mail address: okada@kurims.kyoto-u.ac.jp

ReCeived March 12, 2009 